\title{
An MPL W515L mutation in refractory anemia with ringed sideroblasts associated with marked thrombocytosis: A case report
}

\author{
LIN HAO $^{1}$, SANDEEP SEN $^{2}$ and DHIVYA SUGUMAR ${ }^{1}$ \\ ${ }^{1}$ Department of Internal Medicine; ${ }^{2}$ SSM Cancer Care, Saint Mary's Health Center, St. Louis, MO 63117, USA
}

Received February 16, 2014; Accepted September 26, 2014

DOI: $10.3892 / 01.2014 .2754$

\begin{abstract}
The current study presents the case of a 63-year-old patient exhibiting refractory anemia with ringed sideroblasts associated with marked thrombocytosis (RARS-T), who was positive for the MPL W515L mutation, but negative for the JAK2 V617F mutation. Following diagnosis, the patient remained asymptomatic for over three years, however, in August 2012, the patient relapsed and was administered with supportive treatment in the form of subcutaneous darbepoetin $\alpha$ at a dose of $300 \mu \mathrm{g} /$ week, which resulted in an increased hemoglobin concentration, allowing the patient to remain transfusion-independent. The MPL W515L mutation has been reported in two previous cases of myelodysplastic/myeloproliferative neoplasms (MDS/MPN) with ringed sideroblasts, however, to the best of our knowledge, the current report is the first to present a case of RARS-T with an MPL W515L mutation. A clinical trial designed to evaluate the efficacy of a targeted agent against the JAK2 V617F mutation is currently ongoing, with the aim of providing a novel therapeutic strategy for treating MDS/MPN patients. As MPL is located upstream of the JAK-STAT signaling pathway, it is a possible therapeutic target in MDS/MPN patients positive for an MPL W515L mutation, but negative for a JAK2 V617F mutation.
\end{abstract}

\section{Introduction}

The 2008 World Health Organization (WHO) Classification of Tumors defines refractory anemia with ring sideroblasts associated with marked thrombocytosis (RARS-T) as a provisional entity, with unclassifiable myelodysplastic/myeloproliferative neoplasm (MDS/MPN) status, as opposed to a confirmed entity (1). Although thrombocytosis is a poor prognostic factor in MDS patients (2), overall, RARS-T patients exhibit a more

Correspondence to: Dr Lin Hao, Department of Internal Medicine, Saint Mary's Health Center, 6420 Clayton Road, St. Louis, MO 63117, USA

E-mail: haolinxa@gmail.com

Key words: refractory anemia with ringed sideroblasts associated with marked thrombocytosis, myelodysplatic/myeloproliferative neoplasm, MPLW515L, prognosis, mutation favorable prognosis. The JAK2 V617F mutation has been identified in $\sim 50 \%$ of RARS-T patients and in only 2/89 cases of typical MDS, indicating that RARS-T should be considered as a JAK2 mutation-associated chronic MPN (3).

RARS-T is characterized by MDS characteristics and $<5 \%$ blasts in the bone marrow, and is differentiated from other diseases by the presence of $\geq 15 \%$ ringed sideroblasts, thrombocytosis and a platelet count of $>450 \times 10^{9} / 1$ (1). Clinical manifestations of RARS-T include symptoms associated with anemia, leucopenia and abnormalities of platelet function and quantity, for example, fatigue, infection, bleeding and/or thrombosis. The International Prognostic Scoring System (IPSS) (4), which is commonly used for MDS, is also applicable as a prognostic tool for RARS-T. The management of RARS-T is largely supportive, including transfusion support in patients exhibiting symptomatic anemia and prophylaxis, and treatment of thromboembolism. Similar to MDS, RARS-T patients exhibiting anemia and low serum erythropoietin levels may benefit from the administration of erythropoiesis-stimulating agents. An ongoing clinical trial is currently studying the efficacy of ruxolitinib, an oral JAK2 inhibitor, in JAK2 mutation-positive RARS-T patients (ClinicalTrials.gov Identifier: NCT01895842; http://clinicaltrials.gov/show/NCT01895842). Furthermore, a case study has recently documented the successful treatment of young RARS-T patients with lenalidomide (5).

MPL (6), a cellular homologue of the oncogene v-mpl, belongs to the hematopoietic cytokine receptor family, which is located upstream of the JAK-STAT signaling pathway. It is reported that the MPL W515L mutation is present in $~ 5 \%$ of idiopathic myelofibrosis patients and $\sim 1 \%$ of essential thrombocythemia patients (7). Previous studies have demonstrated that the MPL W515L mutation is associated with an older age, a lower hemoglobin level and higher platelet counts, however, the association between the mutation and complications, such as thrombosis, is not clear $(8,9)$.

The current report presents the case of an RARS-T patient positive for the MPL W515L mutation, but negative for the JAK2 V617F mutation. To the best of our knowledge, this is the first case study of an MPL W515 mutation in a patient with RARS-T.

\section{Case report}

In August 2012, a 63-year-old female patient was referred to Saint Mary's Health Center (St. Louis, MO, USA) for the 
hematological evaluation of macrocytic anemia and thrombocytosis. A complete blood count (CBC) revealed the following results: Hemoglobin, $7.6 \mathrm{~g} / \mathrm{dl}$ (normal range, 12.0-15.0 g/ dl); hematocrit, 24\% (normal range, 36.1-44.3\%); mean corpuscular volume (MCV), $109 \mathrm{fl}$ (normal range, 80-100 fl); platelet count, $834 \times 10^{9} / 1$ (normal range, $150-400 \times 10^{9} / 1$ ); and a normal white blood cell and differential count $8.2 \times 10^{9} / 1$ (normal range, 4-10 x10 $/ 1$ ). An iron panel demonstrated an elevated ferritin level (214 mg/l; normal range, $12-150 \mathrm{ng} / \mathrm{ml})$ and increased iron saturation ( $>95 \%$; normal range, $15-55 \%$ ). Upon examination, the patient was completely asymptomatic. The patient's prior CBC, from September 2010, demonstrated similar macrocytic anemia (hemoglobin, $9.5 \mathrm{~g} / \mathrm{dl}$; MCV, $102 \mathrm{fl}$ ) and thrombocytosis (platelet count, 701 $10^{9} / 1$ ), however, the patient had not been referred for hematological evaluation at that time.

A bone marrow aspiration and biopsy demonstrated marked erythroid hyperplasia, trilineage dyspoiesis (Fig. 1) and increased ring sideroblasts (Fig. 2) compared with the erythroid precursors; $44 \%$ of the precursors were ring sideroblasts. Cytogenetic and fluorescence in situ hybridization analysis of the patient was positive for an MPL W515L mutation and an isolated chromosome $13 \mathrm{q}$ deletion, however, there was no evidence of a chromosome $5 q$ deletion, JAK2 mutation or BCR-ABL fusion gene. According to the WHO Classification of Tumors (1), the patient was diagnosed with RARS-T, with an MPL W515L mutation, a chromosome 13q deletion and an IPSS Score of 0.5 (intermediate-1 risk).

The patient commenced subcutaneous epoetin $\alpha$ therapy (60,000 units/week) from November 2012, however, following a suboptimal response, epoetin $\alpha$ therapy was terminated and subcutaneous darbepoetin $\alpha$ therapy (300 $\mu \mathrm{g} /$ week) commenced. Furthermore, the patient was administered with $81 \mathrm{mg}$ aspirin per day for the treatment of thrombus prophylaxis. From November 2012 until the writing of this study, the patient's hemoglobin concentration (range, 8.0-10.0 g/dl) and

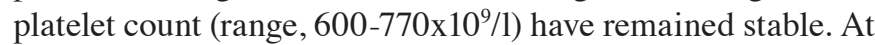
present, the patient is asymptomatic, transfusion-independent, continues to work and maintains a good performance status. A repeat bone marrow biopsy in June 2013 revealed stable hematological results and no evidence of disease progression.

\section{Discussion}

An MPL W515L mutation and isolated chromosome $13 \mathrm{q}$ deletion is rare in an RARS-T patient negative for a JAK2 mutation and $5 q$ deletion. A search of the literature reveals a number of studies regarding MPL W515 mutations in MDS with sideroblastic change, however, it does not reveal any studies on MPL W515 mutations in typical RARS-T. Schnittger et al (10) reported a case of an MPL W515 mutation with features of ringed sideroblasts and thrombocytosis; however, the patient was not anemic and exhibited an asymptomatic benign course. Another study reported the case of a JAK-2 mutation-negative and MPL W515 mutation-positive patient exhibiting grade 2 myelofibrosis; however, no details regarding clinical course, laboratory results or bone marrow biopys results were provided (3).

The JAK-2 V617F mutation occurs in $~ 50 \%$ of RARS-T patients and appears to be predictive of a lower mortality rate

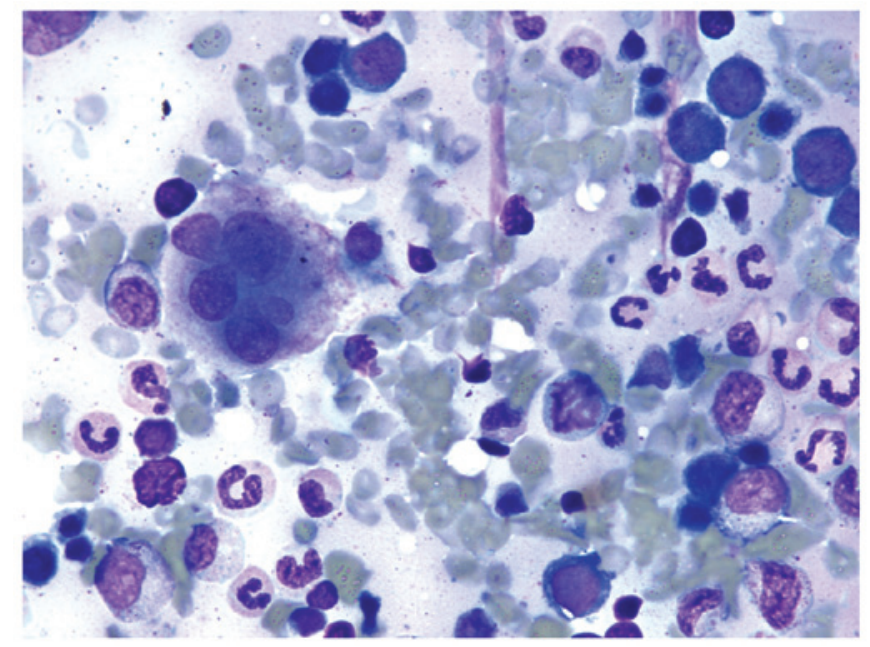

Figure 1. Bone marrow aspirate smear demonstrating hypercellular spicules and trilineage dyspoiesis. Myeloid maturation is mildly dyspoietic and includes hypogranular and hypolobated forms. Erythroid maturation is megaloblastic and megakaryocytes are present in small and monolobated forms (stain, Wright stain; magnification, x1,000).

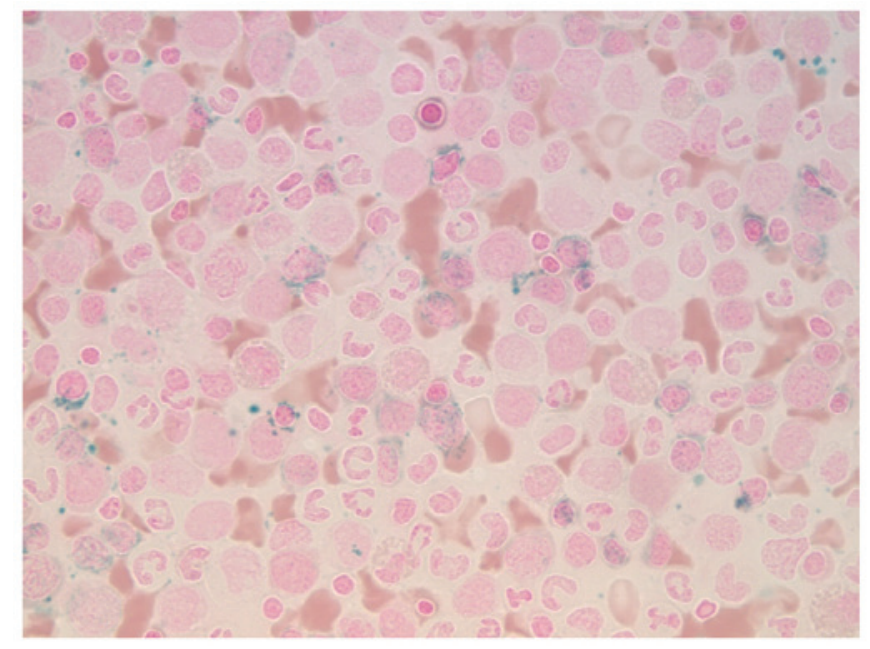

Figure 2. Bone marrow aspirate smear demonstrating an increased number of ring sideroblasts compared with the erythroid precursors (44\% of erythroid precursors were ring sideroblasts; staining, Prussian blue; magnification, x1,000).

compared with the mutation-negative group (3). A clinical trial of ruxolitinib, an oral JAK-2 inhibitor, is currently ongoing with the aim of investigating its efficacy in the treatment of MDS patients who carry the JAK2 V617F mutation (NCT01895842). MPL, a cellular homologue of the v-MPL oncogene, is located upstream of the JAK-STAT signaling pathway (11). The MPL W515L mutation induces constitutive, cytokine-independent activation of the JAK-STAT signaling pathway, and may be significant in the pathogenesis of RARS-T (12). However, it remains unclear whether the MPL W515L mutation is associated with an improved prognosis in RARS-T patients and whether it acts as a target for JAK2 pathway inhibitors, including ruxolitinib. In the case presented in the current study, the patient remained asymptomatic and transfusion-independent with no disease progression at the one year follow-up, indicating that the MPL W515L mutation is associated with a favorable prognosis. However, 
further prospective and long-term investigation of patients exhibiting similar cytogenetic profiles is required prior to reaching a definitive conclusion.

\section{References}

1. Vardiman JW, Bennett JM, Bain BJ, et al: Myelodysplastic/myeloproliferative neoplasm, unclassifiable. In: WHO Classification of Tumours of Haematopoietic and Lymphoid Tissues. Swerdlow SH, Campo E, Harris NL, et al (eds). 4th edition. IARC Press, Lyon, pp85-86, 2008.

2. Zikria J, Galili N, Tsai WY, et al: Thrombocytosis in myelodysplastic syndromes: not an innocent bystander. J Blood Disord Transfus S3: 002, 2012.

3. Schmitt-Graeff AH, Teo SS, Olschewski M, et al: JAK2V617F mutation status identifies subtypes of refractory anemia with ringed sideroblasts associated with marked thrombocytosis. Haematologica 93: 34-40, 2008.

4. Greenberg P, Cox C, LeBeau MM, et al: International scoring system for evaluating prognosis in myelodysplastic syndromes. Blood 89: 2079-2088, 1997.

5. Taylor G, Culligan D and Vickers MA: Refractory anemia with ring sideroblasts associated with marked thrombocytosis complicated by massive splenomegaly treated with lenalidomide resulting in resolution of splenomegaly but severe and prolonged pancytopenia. Case Rep Hemato 2013: 718480,2013
6. Vigon I, Mornon JP, Cocault L, et al: Molecular cloning and characterization of MPL, the human homolog of the v-mpl oncogene: identification of a member of the hematopoietic growth factor receptor superfamily. Proc Natl Acad Sci USA 89: 5640-5644, 1992.

7. Pardanani AD, Levine RL, Lasho T, et al: MPL515 mutations in myeloproliferative and other myeloid disorders: a study of 1182 patients. Blood 108: 3472-3476, 2006.

8. Beer PA, Campbell PJ, Scott LM, et al: MPL mutations in myeloproliferative disorders: analysis of the PT-1 cohort. Blood 112: 141-149, 2008.

9. Vannucchi AM, Antonioli E, Guglielmelli P, et al: Characteristics and clinical correlates of MP $515 \mathrm{~W}>\mathrm{L} / \mathrm{K}$ mutation in essential thrombocythemia. Blood 112: 844-847, 2008.

10. Schnittger S, Bacher U, Haferlach C, et al: Detection of an MPLW515 mutation in a case with features of both essential thrombocythemia and refractory anemia with ringed sideroblasts and thrombocytosis. Leukemia 22: 453-455, 2008.

11. Tefferi A: Novel mutations and their functional and clinical relevance in myeloproliferative neoplasms: JAK2, MPL, TET2, ASXL1, CBL, IDH and IKZF1. Leukemia 24: 1128-1138, 2010.

12. Pikman Y, Lee BH, Mercher T, et al: MPLW515L is a novel somatic activating mutation in myelofibrosis with myeloid metaplasia. PLoS Med 3: e270, 2006. 